\title{
A FINITELY PRESENTED METABELIAN GROUP WITH A FREE ABELIAN DERIVED GROUP OF INFINITE RANK GILBERT BAUMSLAG ${ }^{1}$
}

\begin{abstract}
An example is given of a 3-generator, 3-relator group
\end{abstract} whose derived group is a free abelian group of infinite rank.

1. Very little is known about the normal subgroup structure of finitely presented groups. Indeed it seems to have been fairly widely believed (since 1959, if not before) that an abelian normal subgroup $A$ of a finitely presented group is of finite rank. ${ }^{2}$ The purpose of this note is to put paid to this belief by exhibiting a 3-generator, 3-relator metabelian group $G$ whose derived group $G^{\prime}$ is a free abelian group of infinite rank.

THEOREM. The group

$$
G=\left\langle a, s, t ; s^{t}=s,\left[a^{t}, a\right]=1, a^{s}=a a^{t}\right\rangle
$$

is a metabelian group whose derived group is free abelian of infinite rank.

2. We begin by proving that $G$ is metabelian.

It follows from the defining relations of $G$ that the derived group $G^{\prime}$ of $G$ is generated by the conjugates $a^{t i s^{j}}$ ( $i, j$ arbitrary integers). So it suffices to prove that these conjugates commute. The crux of the proof is the verification that the conjugates $a^{t^{i}}(i=0, \pm 1, \pm 2, \cdots)$ commute. Now $\operatorname{gp}\left(a, a^{t}\right)$ is abelian. Suppose that we have proved that $\operatorname{gp}\left(a, a^{t}, \cdots, a^{t^{n}}\right)$ is abelian for some $n \geqq 1$. Then $\operatorname{gp}\left(a^{t}, a^{t^{2}}, \cdots, a^{t^{n+1}}\right)$ is also abelian. Hence $a^{t}$ commutes with $a, a^{t^{n}}, a^{t^{n+1}}$ and $a^{t^{n}}$ commutes with $a, a^{t}, a^{t^{n+1}}$. Bearing this in mind, and noting the defining relations of $G$, we find

$$
\begin{aligned}
1 & =\left[a, a^{t^{n}}\right]^{s}=\left[a^{s}, a^{t^{n}}\right]=\left[a^{s}, a^{s t^{n}}\right] \\
& =\left[a a^{t},\left(a a^{t}\right)^{t^{n}}\right]=\left[a a^{t}, a^{t^{n}} a^{t^{n+1}}\right]=\left[a, a^{t^{n+1}}\right] .
\end{aligned}
$$

So it follows that $\operatorname{gp}\left(a, a^{t}, \cdots, a^{t^{n+1}}\right)$ is also abelian. But this implies that

$$
\left[a, a^{t^{i}}\right]=1 \quad(i=0, \pm 1, \pm 2, \cdots) .
$$

Received by the editors April 20, 1971.

AMS 1970 subject classifications. Primary 20F05, $20 \mathrm{E} 15$.

Key words and phrases. Finitely presented, metabelian, derived group.

${ }^{1}$ Support from the N.S.F. is gratefully acknowledged.

2 I.e. $A \otimes F$ is finite dimensional over $F$ for every field $F$. 
Now if $j \geqq 0$, then $a^{s^{j}}$ is a product of the elements $a, a^{t}, \cdots, a^{t^{j}}$. Therefore, by (1), $\left[a, a^{t^{i} s^{j}}\right]=1$. If $j<0$, then

$$
1=\left[a^{s^{-j}}, a^{t^{i}}\right]=\left[a^{s^{-j}}, a^{t^{i}}\right]^{s^{j}}=\left[a, a^{t^{i} s^{j}}\right]
$$

again by making use of (1). Thus we have proved that $G^{\prime}$ is abelian.

3. It remains only to show that $G^{\prime}$ is free abelian of infinite rank. To do so, let $F$ be the quotient field of $Z[x]$, the polynomial ring over the integers $Z$ in a single variable $x$. Then the $(2 \times 2)$-matrices over $F$,

$$
\alpha=\left(\begin{array}{ll}
1 & 1 \\
0 & 1
\end{array}\right), \quad \sigma=\left(\begin{array}{cc}
1+x & 0 \\
0 & 1
\end{array}\right), \quad \tau=\left(\begin{array}{ll}
x & 0 \\
0 & 1
\end{array}\right),
$$

generate a group $I$, say. It is easy to check that $\sigma^{\tau}=\sigma,\left[\alpha^{\tau}, \alpha\right]=1$ and $\alpha^{\sigma}=\alpha \alpha^{\tau}$. So the mapping $a \mapsto \alpha, s \mapsto \sigma, t \mapsto \tau$ defines a homomorphism $\phi$ of $G$ into $\Gamma$. Now it follows from the defining relations of $G$ that the elements

$$
\cdots, \text { tat }^{-1}, a, t^{-1} a t, \cdots ; s a s^{-1}, s^{2} a s^{-2}, \cdots
$$

suffice to generate $G^{\prime}$. However inspection shows that the elements

$$
\cdots, \tau \alpha \tau^{-1}, \alpha, \tau^{-1} \alpha \tau, \cdots ; \sigma \alpha \sigma^{-1}, \sigma^{2} \alpha \sigma^{-2}, \cdots
$$

freely generate a free abelian subgroup of $\Gamma$. Since $G^{\prime}$ is abelian and the elements of (2) are mapped by $\phi$ onto the corresponding elements in (3), it follows that the elements of (2) freely generate a free abelian group. So $G^{\prime}$ is free abelian of infinite rank.

ACKNOWLEDGEMENT. I would like to thank the referee for some helpful comments concerning the exposition of this proof.

Department of Mathematics, Rice University, Houston, Texas 77001 